\title{
Effect of Lactobacillus plantarum C014 on Innate Immune Response and Disease Resistance against Aeromonas hydrophila in Hybrid Catfish
}

\author{
Sureerat Butprom, Parichat Phumkhachorn, and Pongsak Rattanachaikunsopon \\ Department of Biological Science, Faculty of Science, Ubon Ratchathani University, Ubon Ratchathani 34190, Thailand \\ Correspondence should be addressed to Pongsak Rattanachaikunsopon; rattanachaikunsopon@yahoo.com
}

Received 4 November 2013; Accepted 5 December 2013

Academic Editors: Y. Mu and D. Zhou

Copyright (C) 2013 Sureerat Butprom et al. This is an open access article distributed under the Creative Commons Attribution License, which permits unrestricted use, distribution, and reproduction in any medium, provided the original work is properly cited.

\begin{abstract}
A bacterial strain isolated from intestines of hybrid catfish (Clarias gariepinus Male $\times$ Clarias macrocephalus Female) exhibited an in vitro inhibitory effect on a fish pathogen, Aeromonas hydrophila TISTR 1321. By using the 16S rDNA sequence analysis, it was identified as Lactobacillus plantarum C014. To examine whether L. plantarum C014 had potential for use as an immunostimulant and biocontrol agent in hybrid catfish, the fish diet supplemented with L. plantarum C014 (10 $\mathrm{CFU} / \mathrm{g}$ diet) was prepared and used for the in vivo investigation of its effect on innate immune response and disease resistance of hybrid catfish. Two innate immune response parameters, phagocytic activity of blood leukocytes and plasma lysozyme activity, were significantly enhanced in the treated fish after 45 days of feeding. Feeding the fish with the L. plantarum C014 supplemented diet for 45 days before challenging them with $A$. hydrophila at the dose of $\mathrm{LD}_{50}$ could reduce the mortality rate of the fish from $50 \%$ (in control group) to $0 \%$ (in treated group). Based on its origin and beneficial effect on innate immune response and disease resistance, L. plantarum C014 may be a potential candidate for use as a natural and safe immunostimulant and biocontrol agent in hybrid catfish.
\end{abstract}

\section{Introduction}

Hybrid catfish (Clarias gariepinus Male $\times$ Clarias macrocephalus Female) has become one of the most important protein sources for Thai people because of its low cost, rapid growth, high availability, and high nutritional value. To match the increasing demand of hybrid catfish, the production of hybrid catfish in Thailand has dramatically increased every year for more than ten years. However, hybrid catfish grown in the intensive aquaculture zare often exposed to stressful conditions which have a negative impact on growth and immunity of the cultured fish. Therefore, hybrid catfish in such environments usually has low growth rate and high tendency to develop diseases, especially bacterial infectious diseases. Among bacteria identified as pathogens in hybrid catfish aquaculture, Aeromonas hydrophila is considered to be one of the foremost economically important pathogens because its infection can lead to growth reduction and unmarketable appearance of infected fish [1].
Currently, bacterial infections in aquaculture, including A. hydrophila infection, are mainly controlled by antibiotics. However, recently, the use of antibiotics in aquaculture has received considerable attention because their use can lead to the development of drug resistant bacteria, thereby reducing drug efficacy. Drug resistance in fish pathogens may also transfer to environmental and human pathogenic bacteria [2]. Moreover, residues of drugs in fish can be potentially risky to consumers and the environment [3]. Therefore, the demand for safer alternative approaches to control the infections has been increasing. One of the most promising methods is to strengthen the defense mechanisms of fish through prophylactic administration of natural immunostimulants. These agents are well known to increase resistance to infectious diseases by enhancing innate (nonspecific) immunity [4]. Among natural immunostimulants, lactic acid bacteria (LAB), especially from fish gastrointestinal tracts, have become potential candidates to replace antibiotics for controlling diseases in fish due to their generally recognized 
as safe (GRAS) status and participation as key components in fish immune response. The relationship between normal endogenous microbiota and the innate immune system of fish was reviewed by Gómez and Balcázar [5]. Several research works have successfully utilized LAB to enhance fish immunity [6-8] and disease resistance ability [9-14]. The beneficial properties of $\mathrm{LAB}$ have brought them to our attention as biocontrol agents against $A$. hydrophila in hybrid catfish.

To obtain a strain of LAB that is suitable for being used as a biocontrol agent in hybrid catfish aquaculture, this study was carried out to isolate a LAB strain from hybrid catfish intestinal tract and evaluate its influence on innate immune response and resistance to $A$. hydrophila infection of the fish.

\section{Materials and Methods}

2.1. Isolation of $L A B$ from Hybrid Catfish Intestines. The intestines were aseptically taken from healthy hybrid catfish (Clarias gariepinus Male $\times$ Clarias macrocephalus Female) cultured at Ubon Ratchathani Rajabhat University, Ubon Ratchathani, Thailand. They were cut into small pieces and mixed with de Man, Rogosa, and Sharpe (MRS) broth to obtain a final concentration of $0.1 \%(\mathrm{w} / \mathrm{v})$. The mixture was incubated at $30^{\circ} \mathrm{C}$ for $24 \mathrm{~h}$ and then centrifuged at $3,000 \times \mathrm{g}$ for $3 \mathrm{~min}$ to remove the fish intestinal residue. One hundred $\mathrm{mL}$ of the supernatant was spreading onto MRS Agar and incubated at $30^{\circ} \mathrm{C}$ for $24 \mathrm{~h}$. Twenty-five isolated LAB colonies grown on the MRS agar were randomly selected for the determination of their antimicrobial activity against a fish pathogenic bacterium A. hydrophila TISTR 1321 obtained from the Institute of Scientific and Technological Research, Ministry of Science and Technology, Thailand.

2.2. Determination of Antimicrobial Activity. Lactic acid bacteria isolated from fish intestines were determined for their antimicrobial activity against the fish pathogen $A$. hydrophila by using agar spot test. Five $\mu \mathrm{L}$ of the tested $\mathrm{LAB}$ cultures grown overnight in MRS broth were spotted onto MRS agar and incubated at $30^{\circ} \mathrm{C}$ for $24 \mathrm{~h}$. Then $5 \mathrm{~mL}$ of the soft $(0.6 \%$ agar) Tryptic Soy Agar (TSA) containing log phase cells of A. hydrophila (at the final concentration of $10^{6} \mathrm{CFU} / \mathrm{mL}$ ) was poured over the agar, previously inoculated with the tested LAB. After incubation at $30^{\circ} \mathrm{C}$ for $24 \mathrm{~h}$, the presence of inhibition zone around the inoculated LAB was examined.

2.3. Identification of $L A B$. The LAB strain showing antimicrobial activity against $A$. hydrophila was identified by Gram staining, morphological observation under light microscope, standard biochemical tests, and 16S rDNA sequence analysis. The $16 \mathrm{~S}$ rDNA sequence analysis was performed as follows. Bacterial genomic DNA used as template DNA for PCR amplification of $16 \mathrm{~S}$ rDNA was extracted from the bacterium by using the Genomic DNA Extraction Kit (Real Biotech Corporation, Taipei, Taiwan) according to the manufacturer's recommendations. The PCR amplification of 16S rDNA was performed using two universal primers, fd1 ( $5^{\prime}$ AGAGTTTGATCCTGGCTCAG $3^{\prime}$ ) [15], and 1492r (5' ACGGCTACCTTGTTACGACTT $3^{\prime}$ ) [16]. The cycling protocol used in this study was $97^{\circ} \mathrm{C}$ for $5 \mathrm{~min}$ followed by 30 cycles of $97^{\circ} \mathrm{C}$ for $45 \mathrm{~s}, 55^{\circ} \mathrm{C}$ for $45 \mathrm{~s}$, and $72^{\circ} \mathrm{C}$ for $90 \mathrm{~s}$. The PCR product was purified and sequenced by BioDesign Co., Ltd. (Pathumthani, Thailand). The sequence of the 16S rDNA was compared with the $16 \mathrm{~S}$ rDNA sequences in the GenBank database using the NCBI Blast program.

2.4. Fish Preparation. Healthy hybrid catfish (Clarias gariepinus Male $\times$ Clarias macrocephalus Female) were obtained from local farms in Ubon Ratchathnai Province, Thailand. To acclimate to laboratory conditions, fish were maintained in $2 \mathrm{~m} \times 2 \mathrm{~m} \times 0.6 \mathrm{~m}$ cement ponds for two weeks prior to experiments. On the day of stocking, the fish were fed $3 \%$ body weight twice a day with the commercial pellet fish diet for small catfish (Charoen Pokphand Group, Bangkok, Thailand). Twenty-four $h$ prior to the experiments, fish weighing $50 \pm 1 \mathrm{~g}$ were transferred from the stocking ponds to $180 \mathrm{~cm} \times 60 \mathrm{~cm} \times 60 \mathrm{~cm}$ aquaria (20 fish per aquarium). Aquaria were cleaned every third day by siphoning off twothirds of water and replacing it with fresh water.

2.5. Preparation of LAB Supplemented Fish Diet. The isolated LAB used in this experiment was Lactobacillus plantarum C014 due to its possession of antimicrobial activity. Its stock suspension was prepared by suspending the LAB cells collected from $24 \mathrm{~h}$ culture of the bacterium in MRS broth to obtain a concentration of $10^{10} \mathrm{CFU} / \mathrm{mL}$.

To prepare the fish diet supplemented with L. plantarum C014 at the final concentration of $10^{7} \mathrm{CFU} / \mathrm{g}$ diet, the bacterial stock suspension was sprayed into the commercial pellet fish diet for small catfish slowly, mixing part by part in a drum mixer. The mixture was passed through a mincedmeat processing machine, producing extruded strings, which were dried at $30^{\circ} \mathrm{C}$ for $24 \mathrm{~h}$ and then broken down to about $2 \mathrm{~mm}$ long pellets. The diet was transferred to plastic bags and stored in a freezer $\left(-20^{\circ} \mathrm{C}\right)$ until used. The L. plantarum C014 supplemented fish diet preparation was repeated every two weeks. Viability of the incorporated L. plantarum C014 bacterial cells in the diet was assessed by bacterial isolation on Lactobacillus selective agar (NEOGEN, Lansing, MI, USA). The isolated bacterial colonies grown on the medium were randomly selected to confirm their identities by using the API $50 \mathrm{CH}$ system (BioMerieux Industry, Hazelwood, MO, USA). For the control diet, it was prepared using the same process as the L. plantarum C014 supplemented diet except adding the same amount of sterile physiological saline to the commercial pellet fish diet instead of L. plantarum C014 suspension.

2.6. Preparation of Leukocytes and Plasma. To study the effect of L. plantarum C014 supplemented fish diet on innate immunity of hybrid catfish, groups of 20 uninfected fish were fed the diets with and without L. plantarum C014 separately. Each feeding was performed in three replicates. Blood samples (4 fish at a time in each group) from caudal vein were collected at $0,15,30,45$, and 60 days after feeding and kept separately in heparin coated plastic tubes (BD, Franklin Lakes, NJ, USA). Individual fish was sampled only once to avoid the influence on the assays due to multiple bleeding 
and handling stress on the fish. Leukocytes and plasma were prepared from each blood sample by using EZ Lympho-SepLymphocyte Separation Tubes (Biological Industries, Kibbutz Beit-Haemek, Israel). Plasma was stored at $-20^{\circ} \mathrm{C}$ until used for analysis. Separated leukocytes were washed twice in Hanks Balanced Salt Solution (HBSS) (Sigma-Aldrich, St. Louis, MO, USA) and resuspended in the same solution to obtain the concentration of $10^{7}$ cells $/ \mathrm{mL}$. The obtained leukocytes and plasma were used for the determination of phagocytic activity and lysozyme activity, respectively. Both activities are parameters of innate immunity.

2.7. Phagocytic Activity Assay. Phagocytic activity of blood leukocytes was determined by using the method of Seeley et al. [17] with some modification. One $\mathrm{mL}$ of leukocyte suspension was mixed with $2 \mathrm{~mL}$ of the Congo red-stained Saccharomyces cerevisiae cell suspension (providing a yeast cell: leukocyte ratio of $20: 1$ ). The mixtures were incubated at room temperature for $1 \mathrm{~h}$. Following incubation, $1 \mathrm{~mL}$ of ice-cold HBSS was added and $1 \mathrm{~mL}$ of histopaque (1.077) was injected into the bottom of each sample tube. The samples were centrifuge at $850 \times \mathrm{g}$ for $5 \mathrm{~min}$ to separate leukocytes from yeast cells. Leukocytes were harvested and washed twice in HBSS. The cells were then resuspended in $1 \mathrm{~mL}$ trypsinEDTA solution $(5.0 \mathrm{~g} / \mathrm{L}$ trypsin and $2.0 \mathrm{~g} / \mathrm{L}$ EDTA, SigmaAldrich) and incubated at $37^{\circ} \mathrm{C}$ overnight. The absorbance of the samples was measured at $510 \mathrm{~nm}$ using trypsin-EDTA as a blank.

Congo red-stained $S$. cerevisiae cells were prepared as follows. Three $\mathrm{mL}$ of a $0.87 \%(\mathrm{w} / \mathrm{v})$ Congo red in phosphate buffer saline (PBS) pH 7.2 was added to yeast cell suspension $(1.5 \mathrm{~g})$. After incubated at room temperature for $15 \mathrm{~min}$, the mixture was thoroughly mixed with $7 \mathrm{~mL}$ of distilled water. The resulting mixture was autoclaved for $15 \mathrm{~min}$, washed three times in HBSS, and stored at $4^{\circ} \mathrm{C}$ until use. Prior to use, the cell concentration of was adjusted to $10^{8}$ cells $/ \mathrm{mL}$ in HBSS.

2.8. Lysozyme Activity Assay. Plasma lysozyme activity was measured using the method of Ellis [18] with some modifications. Briefly, a volume of $0.02 \%(\mathrm{w} / \mathrm{v})$ suspension of Micrococcus lysodeikticus ATCC No. 4698 (Sigma-Aldrich) prepared in $0.05 \mathrm{M}$ phosphate buffer ( $\mathrm{pH}$ 6.2) was used as a substrate. Lyophilized hen egg white lysozyme (SigmaAldrich) was used as a standard. One hundred $\mathrm{mL}$ of standard solutions and fish plasma were added to a $3 \mathrm{~mL}$ suspension of M. lysodeikticus. After incubation for $5 \mathrm{~min}$ at $25^{\circ} \mathrm{C}$, the absorbance of the mixtures was examined at the wavelength of $550 \mathrm{~nm}$. The lysozyme activity of fish plasma was reported as $\mathrm{mg} / \mathrm{mL}$ equivalent of hen egg white lysozyme activity.

2.9. Determination of Median Lethal Dose $\left(L D_{50}\right)$ of $A$. hydrophila. An overnight culture of $A$. hydrophila TISTR 1321 in TSB was centrifuged at 5,000 rpm for 10 minutes. Bacterial cells were washed twice with physiological saline $(0.85 \%$ $\mathrm{NaCl}$ ) and then resuspended in the same solution to obtain a bacterial suspension with the concentration of $10^{8} \mathrm{CFU} / \mathrm{mL}$. The bacterial suspension was subjected to tenfold serial dilutions using the physical saline as a diluent and then used to challenge groups of 20 hybrid catfish. One hundred $\mu \mathrm{L}$ of each dilution was injected intraperitoneally into each fish. For control, the same volume of physiological saline was used instead of the bacterial suspension. Each dilution trial was performed in three replicates. Mortalities were recorded daily for 2 weeks. Dead fish were removed from the aquaria daily. Livers and kidneys were aseptically streaked on Aeromonas Medium Base (Oxoid Limited, Hampshire, UK), a selective medium for $A$. hydrophila. After incubation at $30^{\circ} \mathrm{C}$ for $24 \mathrm{~h}$, colonies grown on the agar were confirmed to be $A$. hydrophila by using the API 20 NE test kit (BioMerieux Industry, Hazelwood, MO, USA). The $\mathrm{LD}_{50}$ value was calculated by the method described by Reed and Muench [19].

\subsection{Test for Effect of L. plantarum C014 Supplemented Fish} Diet on Disease Resistance. Groups of 20 uninfected hybrid catfish with three replicates per each were fed the diets with and without L. plantarum C014 separately for 45 days. After that, fish were challenged intraperitoneally with $0.1 \mathrm{~mL}$ of A. hydrophila TISTR 1321 suspension at a dose causing 50\% mortality $\left(\mathrm{LD}_{50}\right)$. The diets were continued for $14 \mathrm{~d}$ after infection. The mortality in each group was recorded daily. Dead fish were removed from the aquaria daily, and their livers and kidneys were subjected to bacterial isolation on Aeromonas Medium Base. After incubation at $30^{\circ} \mathrm{C}$ for $24 \mathrm{~h}$, the bacteria grown on the medium were identified by using the API 20 NE test kit.

2.11. Statistical Analysis. Analysis of variance (ANOVA) was performed using the general linear models procedure of Statistical Analysis System (SAS Institute, Cary, NC, USA). Duncan's new multiple range test was used to obtain pairwise comparisons among sample means. Evaluations were based on a $5 \%$ significance level $(P<0.05)$.

\section{Results}

Among hundreds of LAB colonies isolated from healthy hybrid catfish's intestines, 25 colonies were randomly selected to examine their antimicrobial activity against a fish pathogen, A. hydrophila TISTR 1321. By using agar spot test, of all 25 LAB isolates, only one isolate, designated as C014, exhibited inhibitory effect on A. hydrophila, indicated by its ability to produce a clear zone against the lawn of $A$. hydrophila.

According to the morphological and biochemical characterization, the LAB isolate C014 was Gram-positive, catalasenegative bacillus. It was unable to produce ammonia from arginine or carbon dioxide from glucose. It was found to grow at $15^{\circ} \mathrm{C}$ but not at $45^{\circ} \mathrm{C}$. The molecular identification of the LAB isolate C014 was carried out by using $16 \mathrm{~S}$ rDNA sequence analysis. The $16 \mathrm{~S}$ rDNA amplified from the LAB using universal primers (fdl and 1492r) comprised about $1,500 \mathrm{bp}$. When the sequence of this amplicon was compared to the known bacterial 16S rDNA sequences in GenBank database using Blast program, it showed 99\% homology to $16 \mathrm{~S}$ rDNA sequence of Lactobacillus plantarum WCFS1 
TABLE 1: Mortality of hybrid catfish in the first 2 weeks after intraperitoneal injection of different dilutions of $A$. hydrophila suspension.

\begin{tabular}{|c|c|c|c|c|c|}
\hline \multicolumn{2}{|c|}{ A. hydrophila } & \multicolumn{3}{|c|}{ No. of dead fish/no. of tested fish ${ }^{a}$} & \multirow{2}{*}{$\%$ Mortality } \\
\hline Concentration (CFU/mL) & Dilution & 1 & 2 & 3 & \\
\hline $10^{7}$ & $10^{-1}$ & $17 / 20$ & $18 / 20$ & $17 / 20$ & 86.67 \\
\hline $10^{6}$ & $10^{-2}$ & $13 / 20$ & $15 / 20$ & $15 / 20$ & 71.67 \\
\hline $10^{5}$ & $10^{-3}$ & $10 / 20$ & $11 / 20$ & $12 / 20$ & 55.00 \\
\hline $10^{4}$ & $10^{-4}$ & $6 / 20$ & $7 / 20$ & $9 / 20$ & 36.67 \\
\hline $10^{3}$ & $10^{-5}$ & $4 / 20$ & $3 / 20$ & $4 / 20$ & 18.37 \\
\hline $10^{2}$ & $10^{-6}$ & $2 / 20$ & $1 / 20$ & $3 / 20$ & 10.00 \\
\hline
\end{tabular}

${ }^{a}$ results from all replicates (replicate 1 to replicate 3 ).

TABLE 2: Mortality of A. hydrophila infected hybrid catfish fed diets with and without L. plantarum C014.

\begin{tabular}{lcc}
\hline Replicate & \multicolumn{2}{c}{ Mortality (\%) } \\
\hline 1 & $\mathrm{~T}$ & $\mathrm{C}$ \\
2 & 50 & 0 \\
3 & 50 & 0 \\
\hline
\end{tabular}

T: Treatment group fed the diet having L. plantarum C014.

C: Control group fed the diet without L. plantarum C014.

(accession NR 075041.1). Thus, the LAB isolate C014 was designated as Lactobacillus plantarum C014.

L. plantarum C014 was selected for further in vivo evaluation of its effect on innate immune response (phagocytic activity and lysozyme activity) and resistance to $A$. hydrophila infection of hybrid catfish because it was the only isolated strain having antimicrobial activity against $A$. hydrophila TISTR 1321.

In the first 30 days of feeding, no significant difference was observed between the phagocytic activity of leukocytes obtained from L. plantarum C014 treated fish and that obtained from the control fish. At day 45 of feeding, the increase of the phagocytic activity of leukocytes was detected in the treatment group but not in the control group. However, no further increase of the phagocytic activity of leukocytes was found in both treatment and control group after day 45 of feeding (Figure 1).

The lysozyme activity of the treatment group was not significantly different from that of the control group for the first 30 days of feeding. However, the difference of this activity between the L. plantarum C014 treated and control fish was initially observed 45 days after feeding. Further feeding of $L$. plantarum C014 supplemented fish diet did not increase the lysozyme activity of the treatment group of fish (Figure 2).

Before we investigated the effect of L. plantarum C014 supplemented fish diet on resistance of hybrid catfish to the disease caused by $A$. hydrophila, the median lethal dose $\left(\mathrm{LD}_{50}\right)$ of the pathogen for hybrid catfish was determined. The mortality of hybrid catfish in the first 2 weeks after intraperitoneal injection of different dilutions of $A$. hydrophila suspension is shown in Table 1 . All of dead fish died within 5 days after bacterial injections and the pathogen was found in

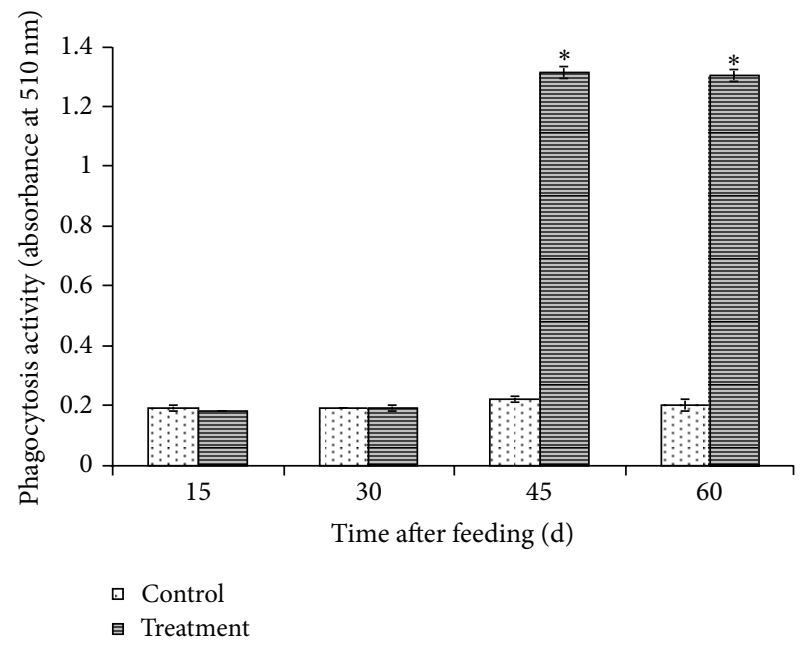

FIGURE 1: Phagocytic activity of isolated leukocytes of hybrid catfish fed diets with (treatment group) and without (control group) $L$. plantarum C014. Results were presented as means \pm SD of triplicate observations. Significant differences $(P<0.05)$ from the control group of the same day are indicated by asterisks.

their livers and kidneys. Based on the mortality, the calculated $\mathrm{LD}_{50}$ of $A$. hydrophila for catfish was $1.07 \times 10^{2} \mathrm{CFU} / \mathrm{g}$ fish.

The effect of the diet supplemented with $L$. plantarum C014 on the resistance of hybrid catfish to A. hydrophila infection was assessed by challenging the fish receiving the diet for 45 days with $A$. hydrophila at the dose of $\mathrm{LD}_{50}$. For the control group, the bacterial pathogen was given to the fish fed with the diet without L. plantarum C014 supplement. The results clearly showed that the L. plantarum C014 was able to prevent the fish from death caused by $A$. hydrophila indicated by the significant decrease of mortality rate from $50 \%$ (in the control fish) to $0 \%$ (in the L. plantarum C014 treated fish) (Table 2).

\section{Discussion}

In fish, the defense against invading pathogens relies mainly on the innate immune system [20]. It consists of 3 major elements including physical barriers, humoral and cellular components. Innate humoral components include antimicrobial peptides, lysozyme, complement, transferrin, pentraxins, 


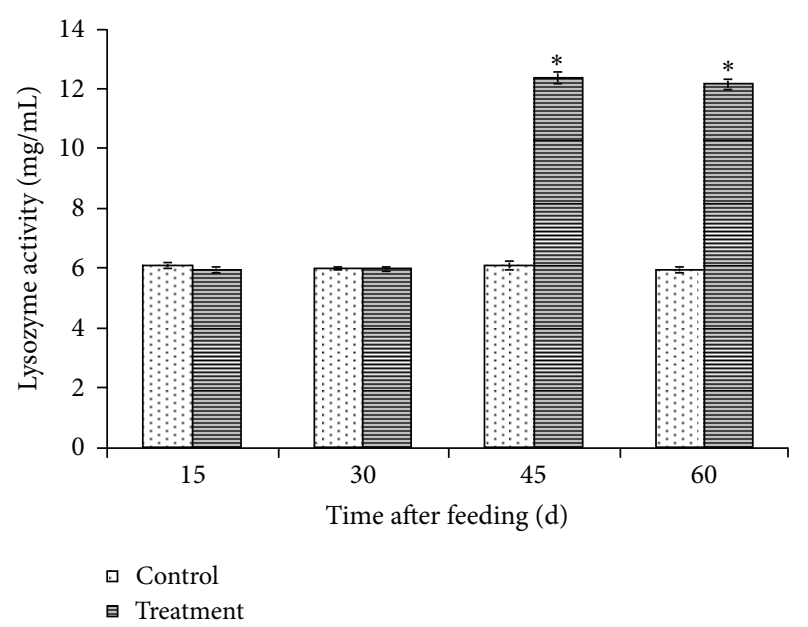

FIGURE 2: Lysozyme activity in hybrid catfish fed diets with (treatment group) and without (control group) L. plantarum C014. Results were presented as means \pm SD of triplicate observations. Significant differences $(P<0.05)$ from the control group of the same day are indicated by asterisks.

lectins, antiproteases, and natural antibodies, whereas the predominant role of the innate cellular components is phagocytosis. Therefore, each of these components can be used as a parameter to determine status of innate immune response of fish. In this study, phagocytic activity and lysozyme activity were used to investigate the effect of L. plantarum C014 supplemented fish diet on innate immune response of hybrid catfish. The reason that we selected oral administration over injection method to give L. plantarum C014 to hybrid catfish is because oral administration is noninvasive and produces no stress to fish, compared to injection method. Furthermore, it is considered to be more practical in fish farming than injection.

Leukocytes are one of the most important cells of the innate immune system of fish. Their phagocytic activity is a primitive defense mechanism and important characteristic of the innate immune system [21]. Therefore, innate immune system and defense mechanism of fish can be enhanced by increasing phagocytic activity of fish leukocytes. In this study, the L. plantarum C014 was clearly shown to be a potential immunostimulant in hybrid catfish because fish diet supplemented with the bacterium boosted the phagocytic activity of fish leukocytes after feeding for 45 days. Several bacteria have been reported to be able to enhance phagocytic activity of fish leukocytes including Bacillus circulans PB7 [22], Bacillus clausii [23], Bacillus pumilus [23], Bacillus subtilis [7], and Lactobacillus delbrueckii subsp. lactis [7]. Currently, substantial amounts of research grants and efforts have been spent on studies to reveal how bacteria enhance the phagocytic activity in fish.

Lysozyme is a bactericidal protein hydrolyzing $\beta-1,4-$ linked glycosidic bonds of bacterial cell wall peptidoglycans resulting in cell lysis. It is known to attack mainly Grampositive bacteria as well as Gram-negative bacteria in conjunction with complements [20]. The L. plantarum C014 was shown in the present study to be a stimulant of lysozyme activity in hybrid catfish. Similar result was reported by Panigrahi et al. [6]. They found that the Lactobacillus rhamnosus JCM 1136 had stimulatory effect on lysozyme activity of rainbow trout Oncorhynchus mykiss. Many fish species including tilapia, catfish, rainbow trout have been known to use lysozyme as a part of innate immune system to protect themselves from pathogens. However, some fish such as cod, haddock, pollack, and wolffish have very little or no lysozyme activity in their tissues and body fluids. These fish on the other hand show high chitinase activity in their plasma and various organs [24]. Therefore, it is believed that these fish species may use chitinase instead of lysozyme to defend themselves against pathogens [20].

The in vivo study is imperative to investigate whether or not the L. plantarum C014 improve resistance of hybrid catfish to A. hydrophila infection even though the bacterium was shown to have an inhibitory effect on A. hydrophila in the in vitro study. This is because several bacteria which were found to have antimicrobial activity in vitro did not improve disease resistance in fish. For example, Abd ElRhman et al. [25] reported that Pseudomonas sp. suppressed the growth of Aeromonas salmonicida in vitro but did not reduce the mortality, caused by A. salmonicida infection in Nile tilapia. The present study investigated the possibility of using L. plantarum C014 as a prophylactic agent to control A. hydrophila infection in hybrid catfish. Since both innate immune response parameters used in this study (phagocytic and lysozyme activities) significantly increased after feeding the fish with $L$. plantarum C014 supplemented fish diet for 45 days, the fish were fed the diet for 45 days before challenging the fish with $A$. hydrophila. The cumulative mortality of $A$. hydrophila infected hybrid catfish fed the L. plantarum C014 containing fish diet decreases significantly, compared to that of the control group. Our results suggested that $L$. plantarum C014 not only inhibited $A$. hydrophila in vitro but enhanced resistance to the disease caused by $A$. hydrophila in hybrid catfish as well.

\section{Conclusion}

In conclusion, this study shows that L. plantarum C014 isolated from hybrid catfish's intestines when added to fish diet at the concentration of $10^{7} \mathrm{CFU} / \mathrm{g}$ diet improved innate immune response and disease resistance in hybrid catfish. The diet elevated not only phagocytic activity and lysozyme activity in hybrid catfish, but also the survival rate of $A$. hydrophila infected hybrid catfish. These results suggest that L. plantarum C014 has a potential to be used as a natural and safe immunostimulants and biocontrol agent against $A$. hydrophila in hybrid catfish. However, many interesting issues are still unclear and require further investigations for proper explanation. These include the following. How can L. plantarum $\mathrm{C} 014$ enhance fish innate immune response? Does the antimicrobial activity of $L$. plantarum $\mathrm{C} 014$ or the enhanced innate immunity of fish play an important role in the increase 
of disease resistance or both of them share an equal responsibility? Is the ability of L. plantarum C014 to raise innate immunity and disease resistance fish species specific and bacteria pathogen strain specific? Research works designed to address all of these questions are underway in our laboratory.

\section{References}

[1] U. Na-Nakron, S. Chantsawang, and W. Tamchalannkit, "Response to mass selection for disease resistance in walking catfish, Clarias macrocephalus," Journal of Applied Aquaculture, vol. 4, no. 4, pp. 65-74, 1995.

[2] P. Smith, M. P. Hiney, and O. B. Samuelsen, "Bacterial resistance to antimicrobial agents used in fish farming: a critical evaluation of method and meaning," Annual Review of Fish Diseases, vol. 4, pp. 273-313, 1994.

[3] D. J. Alderman and T. S. Hastings, "Antibiotic use in aquaculture: development of antibiotic resistance-potential for consumer health risks," International Journal of Food Science and Technology, vol. 33, no. 2, pp. 139-155, 1998.

[4] J. Raa, R. Roerstad, and B. Robertson, "The use of immunostimulants to increase resistance of aquatic organisms to microbial infections," in Diseases in Asian Aquaculture I, I. M. Shariff, R. P. Subasinghe, and J. R. Arthur, Eds., pp. 39-50, Fish Health section, Asian Fisheries Society, Manila, Philippines, 1992.

[5] G. D. Gómez and J. L. Balcázar, "A review on the interactions between gut microbiota and innate immunity of fish," FEMS Immunology and Medical Microbiology, vol. 52, no. 2, pp. 145154, 2008.

[6] A. Panigrahi, V. Kiron, T. Kobayashi, J. Puangkaew, S. Satoh, and H. Sugita, "Immune responses in rainbow trout Oncorhynchus mykiss induced by a potential probiotic bacteria Lactobacillus rhamnosus JCM 1136," Veterinary Immunology and Immunopathology, vol. 102, no. 4, pp. 379-388, 2004.

[7] I. Salinas, A. Cuesta, M. Á. Esteban, and J. Meseguer, "Dietary administration of Lactobacillus delbrüeckii and Bacillus subtilis, single or combined, on gilthead seabream cellular innate immune responses," Fish \& Shellfish Immunology, vol. 19, no. 1, pp. 67-77, 2005.

[8] Y.-B. Wang, Z.-Q. Tian, J.-T. Yao, and W.-F. Li, "Effect of probiotics, Enteroccus faecium, on tilapia (Oreochromis niloticus) growth performance and immune response," Aquaculture, vol. 277, no. 3-4, pp. 203-207, 2008.

[9] F. J. Gatesoupe, "Lactic acid bacteria increase the resistance of turbot larvae, Scophthalmus maximus, against pathogenic Vibrio," Aquatic Living Resources, vol. 7, no. 4, pp. 277-282, 1994.

[10] A. Gildberg, A. Johansen, and J. Bogwald, "Growth and survival of Atlantic salmon (Salmo salar) fry given diets supplemented with fish protein hydrolysate and lactic acid bacteria during a challenge trial with Aeromonas salmonicida," Aquaculture, vol. 138, no. 1-4, pp. 23-34, 1995.

[11] A. Gildberg, H. Mikkelsen, E. Sandaker, and E. Ringø, "Probiotic effect of lactic acid bacteria in the feed on growth and survival of fry of Atlantic cod (Gadus morhua)," Hydrobiologia, vol. 352, no. 1-3, pp. 279-285, 1997.

[12] A. Gildberg and H. Mikkelsen, "Effects of supplementing the feed to Atlantic cod (Gadus morhua) fry with lactic acid bacteria and immuno-stimulating peptides during a challenge trial with Vibrio anguillarum," Aquaculture, vol. 167, no. 1-2, pp. 103-113, 1998.
[13] L. Verschuere, G. Rombaut, P. Sorgeloos, and W. Verstraete, "Probiotic bacteria as biological control agents in aquaculture," Microbiology and Molecular Biology Reviews, vol. 64, no. 4, pp. 655-671, 2000.

[14] R. Parthasarathy, V. Ramasubramanian, and D. Ravi, "Effect of probiotic bacteria as a biocontrol agent against disease causing pathogen in Calta calta (Hamilton, 1822)," International Journal of Current Research and Review, vol. 4, no. 19, pp. 15-24, 2012.

[15] W. G. Weisburg, S. M. Barns, D. A. Pelletier, and D. J. Lane, "16S ribosomal DNA amplification for phylogenetic study, Journal of Bacteriology, vol. 173, no. 2, pp. 697-703, 1991.

[16] D. J. Lane, "16S/23S rRNA sequencing," in Nucleic Acid Techniques in Bacterial Systematics, E. Stackebrandt and M. Goodfellow, Eds., pp. 115-175, John Wiley \& Sons, New York, USA, 1991.

[17] K. R. Seeley, P. D. Gillespie, and B. A. Weeks, "A simple technique for the rapid spectrophotometric determination of phagocytosis by fish macrophages," Marine Environmental Research, vol. 30, no. 1, pp. 37-41, 1990.

[18] A. E. Ellis, "Immunity to bacteria in fish," Fish \& Shellfish Immunology, vol. 9, no. 4, pp. 291-308, 1999.

[19] L. J. Reed and H. Muench, "A simple method of estimating fifty per cent endpoints," American Journal of Epidemiology, vol. 27, no. 3, pp. 493-497, 1938.

[20] B. Magnadóttir, "Innate immunity of fish (overview)," Fish \& Shellfish Immunology, vol. 20, no. 2, pp. 137-151, 2006.

[21] S. K. Whyte, "The innate immune response of finfish-a review of current knowledge," Fish \& Shellfish Immunology, vol. 23, no. 6, pp. 1127-1151, 2007.

[22] P. Bandyopadhyay and P. K. Das Mohapatra, "Effect of a probiotic bacterium Bacillus circulans PB7 in the formulated diets: on growth, nutritional quality and immunity of Catla catla (Ham.)," Fish Physiology and Biochemistry, vol. 35, no. 3, pp. 467-478, 2009.

[23] Y.-Z. Sun, H.-L. Yang, R.-L. Ma, and W.-Y. Lin, "Probiotic applications of two dominant gut Bacillus strains with antagonistic activity improved the growth performance and immune responses of grouper Epinephelus coioides," Fish \& Shellfish Immunology, vol. 29, no. 5, pp. 803-809, 2010.

[24] R. Fänge, G. Lundblad, and J. Lind, "Lysozyme and chitinase in blood and lymphomyeloid tissues of marine fish," Marine Biology, vol. 36, no. 3, pp. 277-282, 1976.

[25] A. M. Abd El-Rhman, Y. A. E. Khattab, and A. M. E. Shalaby, "Micrococcus luteus and Pseudomonas species as probiotics for promoting the growth performance and health of Nile tilapia, Oreochromis niloticus," Fish \& Shellfish Immunology, vol. 27, no. 2, pp. 175-180, 2009. 

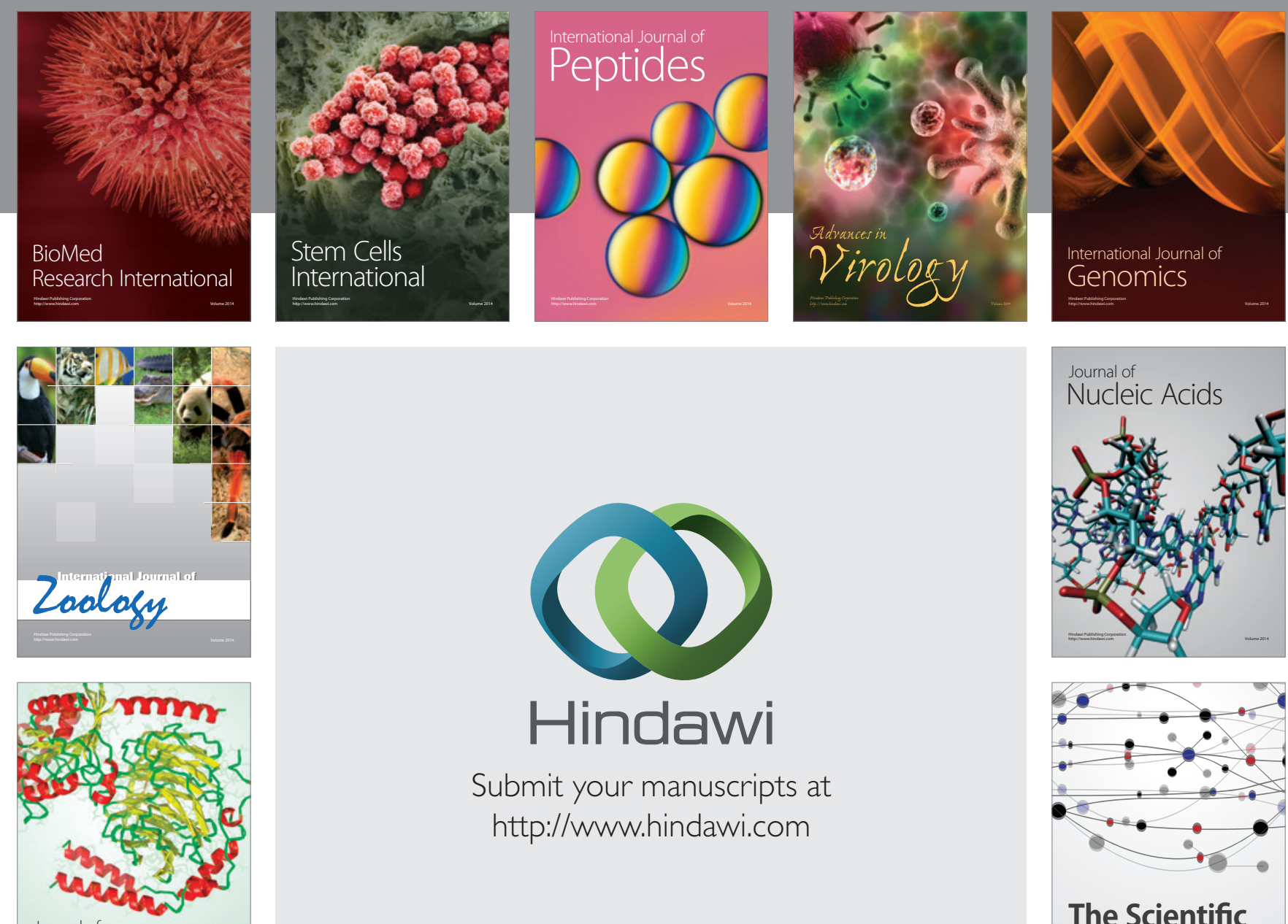

Submit your manuscripts at

http://www.hindawi.com

Journal of
Signal Transduction
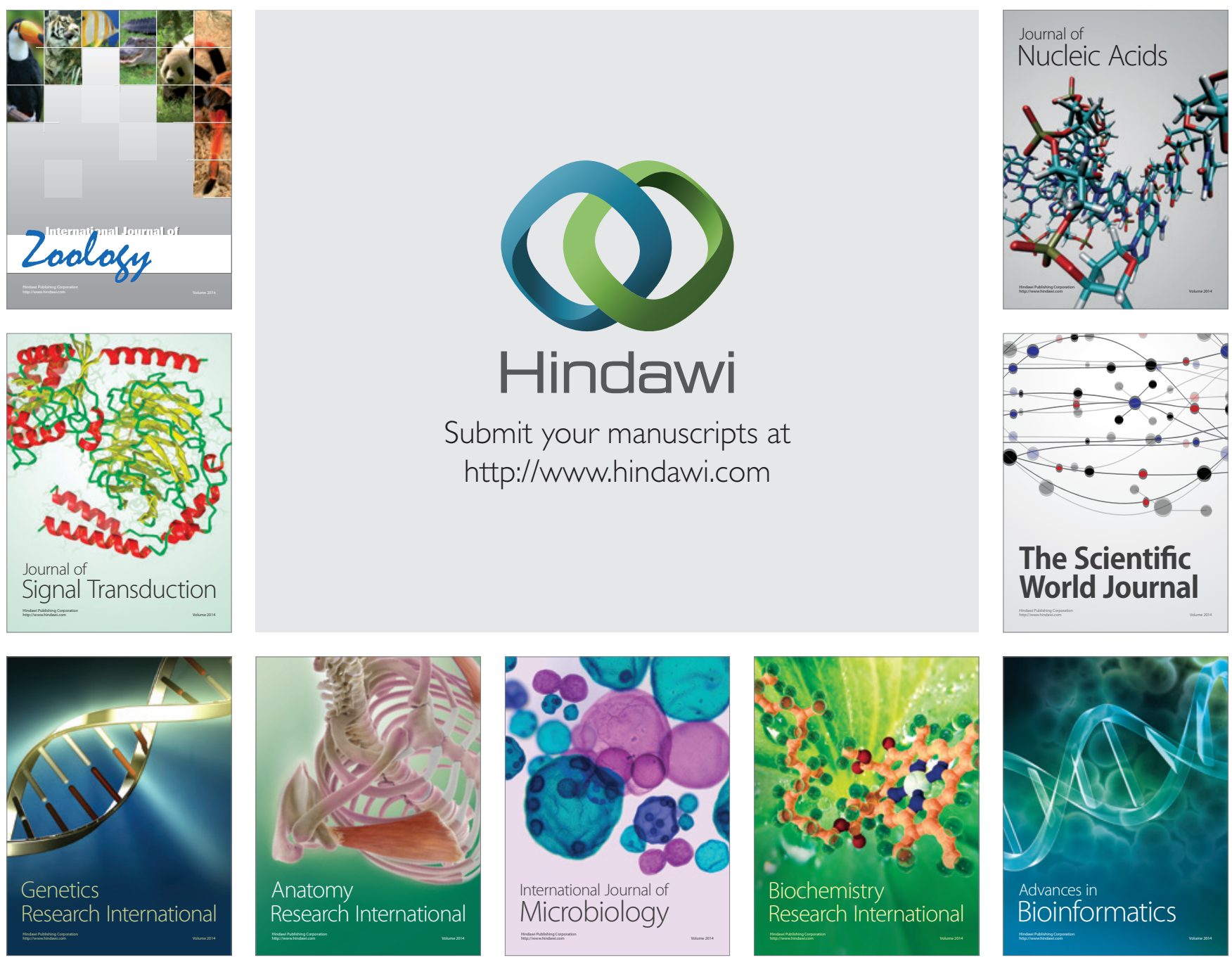

The Scientific World Journal
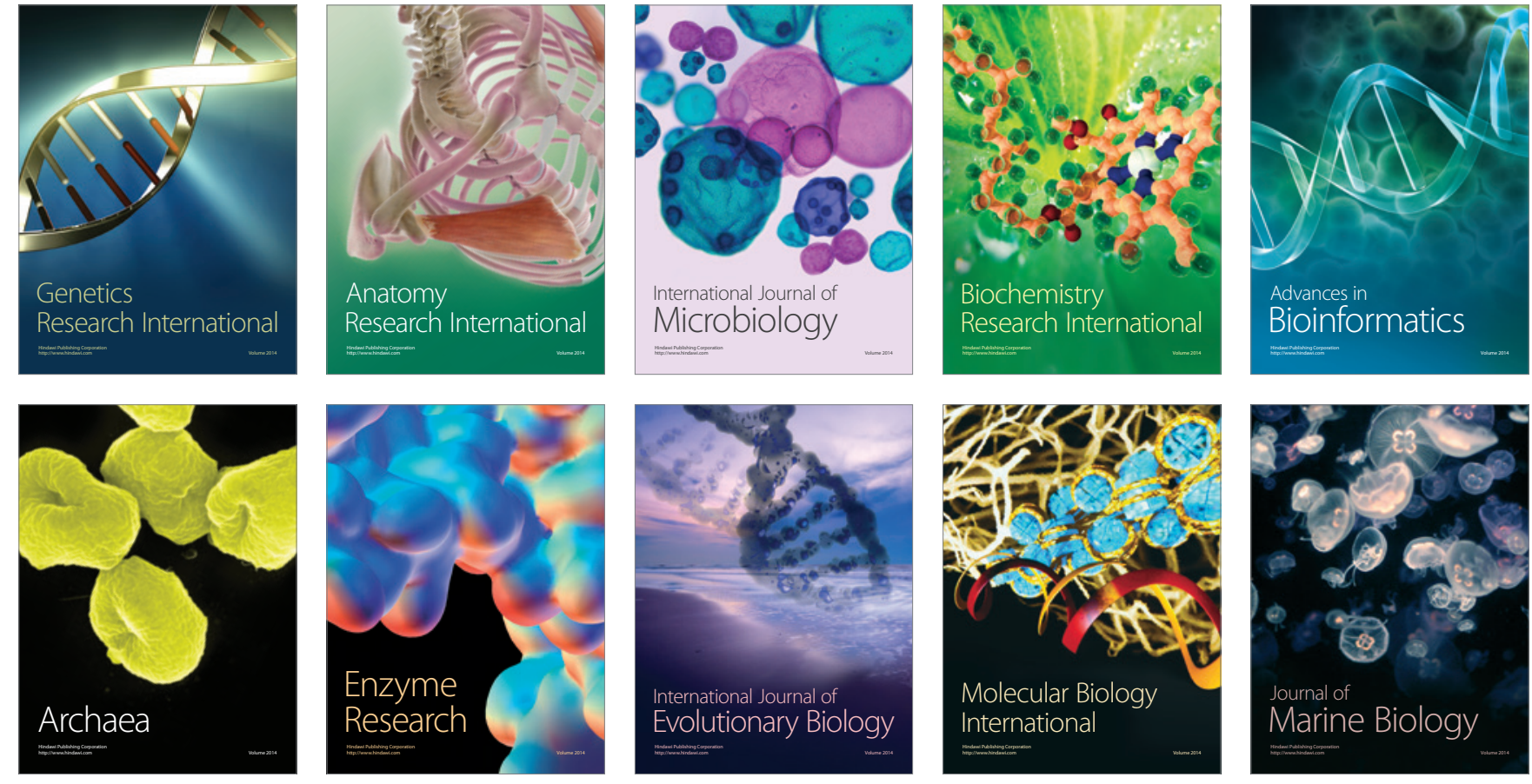